\title{
Leader-Follower Formation Control as a Disturbance Decoupling Problem
}

\author{
Fabio Morbidi, Luca Consolini, Domenico Prattichizzo, Mario Tosques
}

\begin{abstract}
In this paper we present the leader-follower formation control of nonholonomic mobile robots as a disturbance decoupling problem. By means of purely geometrical conditions we show that the formation control problem is solvable if and only if the velocity vector of the leader can be measured and a scalable solution to the problem is provided. The main idea of the work is that of interpreting leader control input as a disturbance affecting the state of the leader-follower dynamic system. Extensive simulation experiments prove the effectiveness of the proposed approach.
\end{abstract}

\section{INTRODUCTION}

A growing interest on coordination and control of multiple autonomous agents matured in the last decade. Their use in several applications such as terrain and utilities inspection, disaster monitoring, search and rescue, structures moving and assembling, space exploration, revealed more and more essential.

For its wide range of applicability, formation control turned out to be one of the most challenging research areas. By formation control we simply mean the problem of controlling the relative position and orientation of the robots in a group while allowing the group to move as a whole. In this field, research dealt with ground vehicles [7], [17], [18], unmanned aerial vehicles (UAVs) [4], [14], aircraft [9], [11], surface and underwater autonomous vehicles (AUVs) [8], [10].

The formation control problem has been tackled using several approaches: behavior based [1], [15], virtual structure [16], leader following [5], [7].

This paper deals with the leader-follower approach. A robot of the formation, designed as the leader, moves along a predefined trajectory while the other robots, the followers, are to maintain a desired posture (distance and orientation) to the leader. Perhaps the main criticism to the leader-follower approach is that the role of the leader is essential to the formation and this affects the reliability of the paradigm. Nonetheless this approach is particularly appreciated because of its flexibility and modularity, especially when new followers join the formation.

F. Morbidi and D. Prattichizzo are with Dipartimento di Ingegneria dell'Informazione, University of Siena, Via Roma 56, 53100 Siena, Italy \{morbidi, prattichizzo\}@di.unisi.it

L. Consolini is with Dipartimento di Ingegneria dell'Informazione, University of Parma, Parco Area delle Scienze 181/a, 43100 Parma, Italy luca.consolini@polirone.mn. it

M. Tosques is with Dipartimento di Ingegneria Civile, University of Parma, Parco Area delle Scienze 181/a, 43100 Parma, Italy mario.tosques@unipr.it
Basile and Marro [2] and independently Wonham and Morse [22], introduced the concept of controlled and conditioned invariant subspaces in the late 1960's, thus inaugurating the geometric approach in linear system theory. Controlled and conditioned invariants are the tools that extend the concept of invariance in linear systems. They make the definition of some system properties (besides controllability and observability) and the solution of some basic problems very straightforward (see [3] for a complete treatment of these topics).

The disturbance decoupling problem (DDP) is a basic problem of the geometric approach, and it was studied by Basile and Marro in their seminal papers as one of the earliest applications of the new invariance concepts. The basic idea is to use feedback in order to make some outputs of the system independent of certain inputs. In particular if an additional input (an undesired perturbation) affects the state of the system, we look for a state feedback with the property that, in the corresponding closed loop system, the perturbation has no influence on the output. If a structural condition is verified the problem admits a solution and suitable algorithms allow the construction of the state feedback control law. In particular when the disturbance is measurable we have a milder solvability condition and a feedforward unit can be applied.

The concept of controlled invariance was extended to general nonlinear systems by Nijmeijer and Van der Schaft [19] in the eighties. In [13], [20] the DDP was reformulated and solved in the nonlinear case using the notion of controlled invariant distribution.

In spite of the power and elegance of the geometric approach, only few problems arising from "non-conventional" application areas have been reformulated and solved as DDPs (one exception is represented for example by [21]). Research indeed has not exhausted yet the potential of the geometric approach for the solution of several interesting control problems.

In this paper we present the leader-follower formation control of nonholonomic mobile robots as a disturbance decoupling problem. To the best of our knowledge this is the first time that a formation control problem is stated in this geometric framework. We show that the formation control problem is solvable if and only if the velocity vector of the leader can be measured. A scalable (with the number of followers) solution to this problem is also provided. The key idea of the paper is to interpret leader control input as a disturbance affecting the state of the leader-follower dynamic system. 
The paper is organized as follows. In Sect. II we introduce the leader-follower setup and we present the formation control problem. In Sect. III we briefly review the DDP for nonlinear systems affine in the control. In Sect. IV we reformulate the leader-follower formation control problem as a DDP with disturbance measurement and we explicitly derive the state feedback control law. Sect. V is devoted to the simulation experiments. In Sect. VI the major contributions of the paper are summarized and future research lines are highlighted.

Notation. The following notation is used through the paper: $\forall x, y \in \mathbb{R}^{n},\langle x, y\rangle=\sum_{i=1}^{n} x_{i} y_{i},\|x\|=\sqrt{\langle x, x\rangle}$.

$\forall x \in \mathbb{R}^{2} \backslash\{0\}, \arg (x)=\theta$, where $\theta \in[0,2 \pi)$ and $x=$ $\|x\|(\cos \theta \quad \sin \theta)^{T}$.

Given $\ell: U \subseteq \mathbb{R}^{n} \rightarrow \mathbb{R}$ and $v: U \subseteq \mathbb{R}^{n} \rightarrow \mathbb{R}^{n}$, $d \ell(x)=\left(\frac{\partial \ell}{\partial x_{1}} \ldots \frac{\partial \ell}{\partial x_{n}}\right), L_{v} \ell(x)=\langle d \ell(x), v(x)\rangle$ and $L_{v}^{r} \ell(x)=\left\langle d L_{v}^{r-1} \ell(x), v(x)\right\rangle$ with $r$ non-negative integer and $L_{v}^{0} \ell(x)=\ell(x)$ by definition.

Given a codistribution $\Omega(x)$, its annihilator is defined as $\Omega^{\perp}(x)=\left\{v \in \mathbb{R}^{n} \mid\left\langle w^{*}, v\right\rangle=0, \forall w^{*} \in \Omega(x)\right\}$.

\section{FORMATION CONTROL}

Fig. 1 shows the leader-follower setup we consider in the paper. It consists of $n+1$ nonholonomic mobile robots: one of the robots is the leader $\left(R_{L}\right)$ while the others are the followers $\left(R_{F_{i}}, i=1,2, \ldots, n\right)$. The kinematics of the leader and the followers is described by the unicycle model

$$
\begin{aligned}
& \dot{x}_{L}=v_{L} \cos \theta_{L} \\
& \dot{y}_{L}=v_{L} \sin \theta_{L} \\
& \dot{\theta}_{L}=\omega_{L}
\end{aligned}
$$

and

$$
\begin{aligned}
\dot{x}_{F_{1}} & =v_{F_{1}} \cos \theta_{F_{1}} \\
\dot{y}_{F_{1}} & =v_{F_{1}} \sin \theta_{F_{1}} \\
\dot{\theta}_{F_{1}} & =\omega_{F_{1}} \\
& \vdots \\
\dot{x}_{F_{n}} & =v_{F_{n}} \cos \theta_{F_{n}} \\
\dot{y}_{F_{n}} & =v_{F_{n}} \sin \theta_{F_{n}} \\
\dot{\theta}_{F_{n}} & =\omega_{F_{n}}
\end{aligned}
$$

where $\left(\begin{array}{ll}x_{L} & y_{L}\end{array}\right),\left(\begin{array}{ll}x_{F_{i}} & y_{F_{i}}\end{array}\right)$, represent the position of the leader and respectively the followers. Analogously $\theta_{L}, \theta_{F_{i}}$ are the orientation of the leader and followers with respect to the reference frame $(x, y)$. Finally $v_{L}, v_{F_{i}}$ and $\omega_{L}, \omega_{F_{i}}$ are the linear and angular velocities of the robots. With reference to Fig. 1, we introduce the following definition [5]:

Definition 1: Set $d_{i}>0$ and $\phi_{i}:\left|\phi_{i}\right|<\frac{\pi}{2}$, $i=1,2, \ldots, n$; the robots $R_{L}$ and $R_{F_{i}}$ make a formation with leader $R_{L}$, if, $\forall t \geq 0, \forall i=1,2, \ldots, n$,

$$
\begin{aligned}
\left\|\mathrm{L}(t)-\mathrm{F}_{i}(t)\right\| & =d_{i} \\
\arg \left(\mathrm{L}(t)-\mathrm{F}_{i}(t)\right)-\theta_{F_{i}}(t) & =\phi_{i}
\end{aligned}
$$

where $\mathrm{L}=\left(\begin{array}{ll}x_{L} & y_{L}\end{array}\right)$ and $\mathrm{F}_{i}=\left(\begin{array}{ll}x_{F_{i}} & y_{F_{i}}\end{array}\right)$.

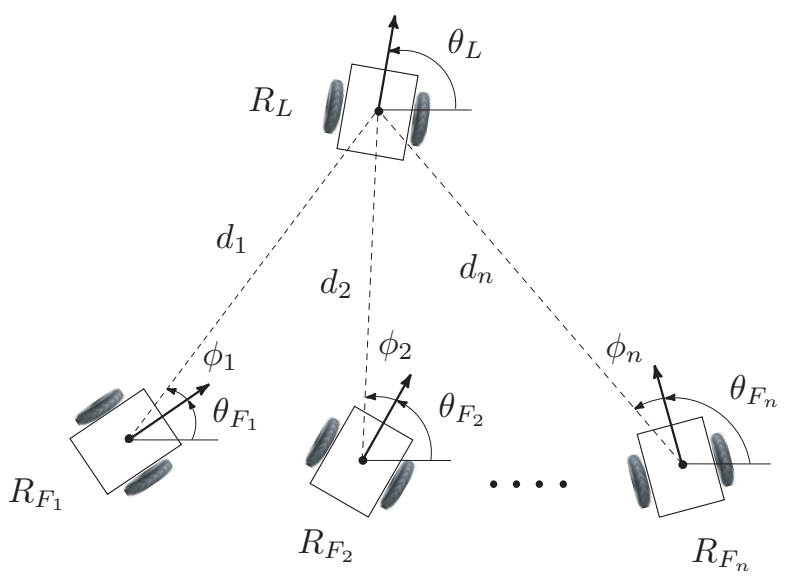

Fig. 1. Leader-follower setup.

Consider the following problem:

Problem 1 (Formation control problem):

Find the control functions $v_{F_{i}}, \omega_{F_{i}}$, such that, starting from arbitrary initial states $\left(x_{L}(0) y_{L}(0)\right),\left(x_{F_{i}}(0) \quad y_{F_{i}}(0)\right)$, equations (3) and (4) are asymptotically satisfied.

\section{DISTURBANCE DECOUPLING}

For the reader convenience we here report the main results on disturbance decoupling for nonlinear systems affine in the control. A complete review of these topics can be found in [12] and references therein.

Consider the following nonlinear system,

$$
\begin{aligned}
\dot{x} & =f(x)+\sum_{k=1}^{m} g_{k}(x) u_{k}+\sum_{j=1}^{q} p_{j}(x) \sigma_{j} \\
y_{1} & =h_{1}(x) \\
& \vdots \\
y_{m} & =h_{m}(x)
\end{aligned}
$$

where $f(x), g_{1}(x), \ldots, g_{m}(x), p_{1}(x), \ldots, p_{q}(x)$ are smooth vector fields and $h_{1}(x), \ldots, h_{m}(x)$ smooth functions defined on an open subset $\mathcal{X}$ of $\mathbb{R}^{s}$. The additional input $\sigma$ represents an undesired perturbation that affects the behavior of the system through $p(x)$.

The disturbance decoupling problem can be formalized as follows [12]:

Problem 2 (Disturbance decoupling problem): Consider a system of the form (5) and a point $x^{o}$. Find, if possible, a static state feedback control

$$
u=\alpha(x)+\beta(x) \mu
$$

defined in a neighborhood of $x^{o}$, with $\mu$ external reference input and $\alpha(x), \beta(x)$ suitable functions, yielding a closedloop system in which the output $y$ is completely independent of, or decoupled from the disturbance $\sigma$.

Let $\Delta^{\star}$ be the largest locally controlled invariant distribution contained in $\operatorname{ker}(d h) \triangleq \bigcap_{k=1}^{m} \operatorname{ker}\left(d h_{k}\right)$, 
$G=\operatorname{span}\left\{g_{1}, \ldots, g_{m}\right\}$ and $P=\operatorname{span}\left\{p_{1}, \ldots, p_{q}\right\}$, where $g_{k}$ and $p_{k}$ are the $k$ th column of $g(x)$ and $p(x)$, respectively.

In general the calculation of $\Delta^{\star}$ is made possibile by a recursive algorithm. However, in some special cases the computation of $\Delta^{\star}$ turns out to be particularly simple.

Proposition 1: Suppose system (5) has vector relative degree $\left\{r_{1}, \ldots, r_{m}\right\}$ at a point $x^{o}$. The largest locally controlled invariant distribution contained in $\operatorname{ker}(d h)$, can be expressed, in a neighborhood of $x^{o}$, as

$$
\Delta^{\star}=\bigcap_{k=1}^{m} \bigcap_{j=1}^{r_{k}} \operatorname{ker}\left(d L_{f}^{j-1} h_{k}\right) \text {. }
$$

Note that if system (5) has relative degree at $x^{o}$, the disturbance decoupling problem amounts to the problem of using feedback in order to constrain the output of the system to be zero for a certain time.

The following proposition [12], [20] provides a necessary and sufficient condition to locally solve Problem 2 .

Proposition 2: Suppose $\Delta^{\star}$ and $\Delta^{\star}+G$ are nonsingular in a neighborhood of $x^{o}$. The disturbance decoupling problem is solvable if and only if

$$
P \subseteq \Delta^{\star}
$$

in a neighborhood of $x^{o}$. If this is the case then a solution is given by (6) with

$$
\begin{aligned}
& \alpha(x)=-A^{-1}(x) b(x) \\
& \beta(x)=A^{-1}(x)
\end{aligned}
$$

where

$$
\begin{gathered}
A(x)=\left(\begin{array}{ccc}
L_{g_{1}} L_{f}^{r_{1}-1} h_{1}(x) & \cdots & L_{g_{m}} L_{f}^{r_{1}-1} h_{1}(x) \\
\vdots & \ddots & \vdots \\
L_{g_{1}} L_{f}^{r_{m}-1} h_{m}(x) & \cdots & L_{g_{m}} L_{f}^{r_{m}-1} h_{m}(x)
\end{array}\right) \\
b(x)=\left(\begin{array}{lllc}
L_{f}^{r_{1}} h_{1}(x) & L_{f}^{r_{2}} h_{2}(x) & \cdots & L_{f}^{r_{m}} h_{m}(x)
\end{array}\right)^{T} .
\end{gathered}
$$

Note that if system (5) has an asymptotically stable zero dynamics, the choice

$$
\mu=-\left(\begin{array}{c}
\lambda_{10} h_{1}(x)+\ldots+\lambda_{1 r_{1}-1} L_{f}^{r_{1}-1} h_{1}(x) \\
\vdots \\
\lambda_{m 0} h_{m}(x)+\ldots+\lambda_{m r_{m}-1} L_{f}^{r_{m}-1} h_{m}(x)
\end{array}\right)
$$

with $\lambda_{k j}$ positive gains, guarantees asymptotic stability.

Sometimes it is possible to obtain measurements of the disturbance and use them in order to design the control law. If the disturbance $\sigma$ is available for measurements, control (6) can be replaced by

$$
u=\alpha(x)+\beta(x) \mu+\gamma(x) \sigma
$$

which, besides to a feedback on $x$, includes a feedforward on the disturbance $\sigma$. Furthermore, decoupling the output from the disturbance is possible under conditions that are obviously weaker than those established in Proposition 2. In fact condition (7) becomes

$$
P \subseteq \Delta^{\star}+G
$$

and the solution of the disturbance decoupling problem with disturbance measurement is given by (10) with $\alpha(x)$ and $\beta(x)$ defined in (8) and

$$
\gamma(x)=-A^{-1}(x) C(x)
$$

where

$$
C(x)=\left(\begin{array}{ccc}
L_{p_{1}} L_{f}^{r_{1}-1} h_{1}(x) & \cdots & L_{p_{q}} L_{f}^{r_{1}-1} h_{1}(x) \\
\vdots & \ddots & \vdots \\
L_{p_{1}} L_{f}^{r_{m}-1} h_{m}(x) & \cdots & L_{p_{q}} L_{f}^{r_{m}-1} h_{m}(x)
\end{array}\right) .
$$

\section{FORMATION CONTROL AS A DISTURBANCE DECOUPLING PROBLEM}

By formulating the formation control problem as a disturbance decoupling problem, in the next proposition we prove that it admits a solution if and only if the leader velocity vector can be measured. The analytical solution of the problem is also derived.

Proposition 3: The formation control problem is solvable if and only if the vector $\left(v_{L} \omega_{L}\right)^{T}$ can be measured. If this is the case, asymptotic stability is guaranteed by the static state feedback control (10), with

$$
\alpha(x)=\left(\begin{array}{lllll}
0 & 0 & \cdots & 0 & 0
\end{array}\right)^{T}
$$

$\beta(x)=\operatorname{diag}\left\{\left(\begin{array}{cc}-\frac{\cos \left(\theta_{F_{1}}+\phi_{1}\right)}{\cos \phi_{1}} & -\frac{\sin \left(\theta_{F_{1}}+\phi_{1}\right)}{\cos \phi_{1}} \\ \frac{\sin \theta_{F_{1}}}{d_{1} \cos \phi_{1}} & -\frac{\cos \theta_{F_{1}}}{d_{1} \cos \phi_{1}}\end{array}\right), \ldots\right.$

$$
\left.\ldots,\left(\begin{array}{cc}
-\frac{\cos \left(\theta_{F_{n}}+\phi_{n}\right)}{\cos \phi_{n}} & -\frac{\sin \left(\theta_{F_{n}}+\phi_{n}\right)}{\cos \phi_{n}} \\
\frac{\sin \theta_{F_{n}}}{d_{n} \cos \phi_{n}} & -\frac{\cos \theta_{F_{n}}}{d_{n} \cos \phi_{n}}
\end{array}\right)\right\}
$$

$$
\gamma(x)=\left(\begin{array}{cc}
\frac{\cos \left(\theta_{L}-\theta_{F_{1}}-\phi_{1}\right)}{\cos \phi_{1}} & 0 \\
\frac{\sin \left(\theta_{L}-\theta_{F_{1}}\right)}{d_{1} \cos \phi_{1}} & 0 \\
\vdots & \vdots \\
\frac{\cos \left(\theta_{L}-\theta_{F_{n}}-\phi_{n}\right)}{\cos \phi_{n}} & 0 \\
\frac{\sin \left(\theta_{L}-\theta_{F_{n}}\right)}{d_{n} \cos \phi_{n}} & 0
\end{array}\right)
$$

and $\mu=-\left(\begin{array}{llll}\lambda_{1} h_{1}(x) & \lambda_{2} h_{2}(x) & \cdots & \lambda_{2 n} h_{2 n}(x)\end{array}\right)^{T}$ with $\lambda_{1}, \lambda_{2}, \ldots, \lambda_{2 n}$ positive constants. 


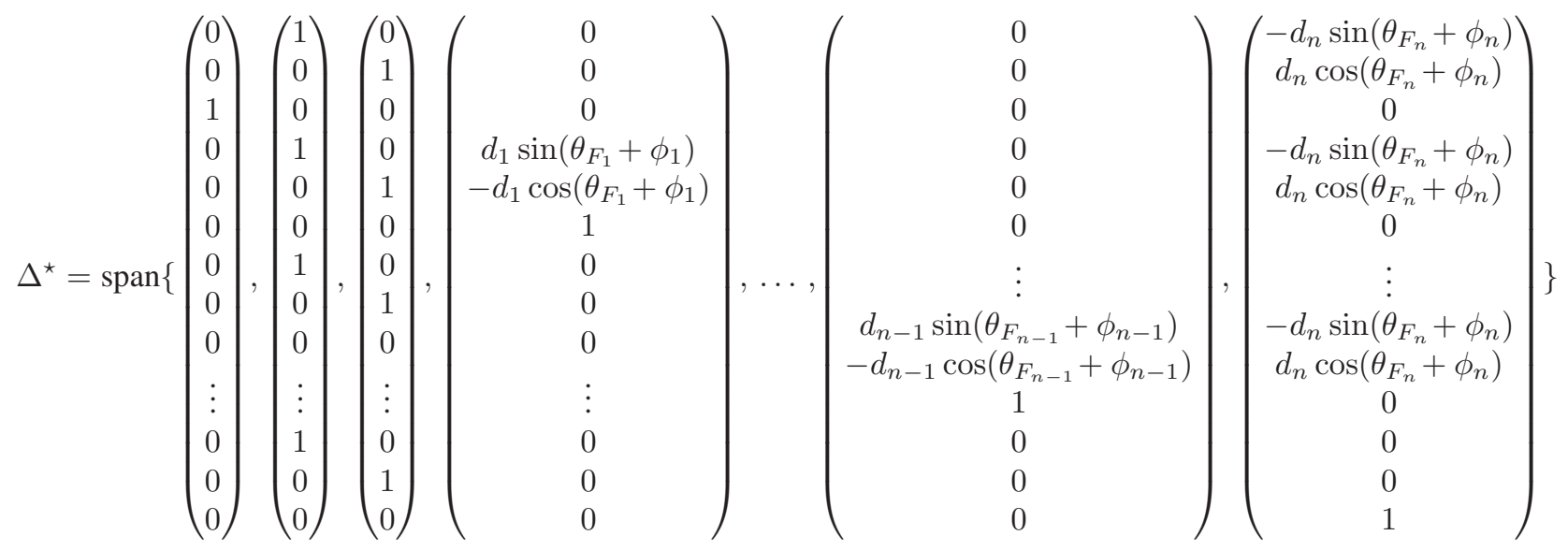

Proof: According to system (5), write equations (1) -(2) as

$$
\dot{x}=\sum_{k=1}^{2 n} g_{k}(x) u_{k}+\sum_{j=1}^{2} p_{j}(x) \sigma_{j}
$$

where

$g(x)=\left(\begin{array}{cclcc}0 & 0 & \cdots & 0 & 0 \\ 0 & 0 & \cdots & 0 & 0 \\ 0 & 0 & \cdots & 0 & 0 \\ \cos \theta_{F_{1}} & 0 & \cdots & 0 & 0 \\ \sin \theta_{F_{1}} & 0 & \cdots & 0 & 0 \\ 0 & 1 & \cdots & 0 & 0 \\ \vdots & \vdots & \ddots & \vdots & \vdots \\ 0 & 0 & \cdots & \cos \theta_{F_{n}} & 0 \\ 0 & 0 & \cdots & \sin \theta_{F_{n}} & 0 \\ 0 & 0 & \cdots & 0 & 1\end{array}\right), p(x)=\left(\begin{array}{cc}\cos \theta_{L} & 0 \\ \sin \theta_{L} & 0 \\ 0 & 1 \\ 0 & 0 \\ 0 & 0 \\ 0 & 0 \\ \vdots & \vdots \\ 0 & 0 \\ 0 & 0 \\ 0 & 0\end{array}\right)$ $x=\left(\begin{array}{llllllllll}x_{L} & y_{L} & \theta_{L} & x_{F_{1}} & y_{F_{1}} & \theta_{F_{1}} & \ldots & x_{F_{n}} & y_{F_{n}} & \theta_{F_{n}}\end{array}\right)^{T} \in \mathcal{X} \subseteq$ $\mathbb{R}^{3(n+1)}, u=\left(\begin{array}{lllll}v_{F_{1}} & \omega_{F_{1}} & \ldots & v_{F_{n}} & \omega_{F_{n}}\end{array}\right)^{T} \in \mathbb{R}^{2 n}$ and $\sigma=\left(\begin{array}{ll}v_{L} & \omega_{L}\end{array}\right)^{T}$ is now supposed to be an undesired input or disturbance. Assume that the output of system (16) is

$$
y=\left(\begin{array}{cc}
\mathrm{L}-\mathrm{F}_{1}-d_{1}\left(\cos \left(\theta_{F_{1}}+\phi_{1}\right)\right. & \left.\sin \left(\theta_{F_{1}}+\phi_{1}\right)\right)^{T} \\
\vdots & \\
\mathrm{L}-\mathrm{F}_{n}-d_{n}\left(\cos \left(\theta_{F_{n}}+\phi_{n}\right)\right. & \left.\sin \left(\theta_{F_{n}}+\phi_{n}\right)\right)^{T}
\end{array}\right) .
$$

Differentiating $y$, we find that under the hypotheses of definition 1 (that guarantee that the decoupling matrix $A(x)$ is nonsingular, in fact $\operatorname{det}(A(x))=$ $\left.d_{1} \cos \phi_{1} d_{2} \cos \phi_{2} \cdots d_{n} \cos \phi_{n}\right)$, system (16)-(17) has relative degree $\{1,1, \ldots, 1\}$ in $\mathcal{X}$.

Therefore, according to Proposition $1, \Delta^{\star}$ can be written as

$$
\Delta^{\star}=\bigcap_{k=1}^{2 n} \operatorname{ker}\left(d h_{k}\right) \triangleq\left(\operatorname{span}\left\{d h_{1}, \ldots, d h_{2 n}\right\}\right)^{\perp}
$$

where $\left(\operatorname{span}\left\{d h_{1}, \ldots, d h_{2 n}\right\}\right)^{\perp}$ is the annihilator of the codistribution $\operatorname{span}\left\{d h_{1}, \ldots, d h_{2 n}\right\}$. The differentials are

$d h_{1}=\left(\begin{array}{lllllllllllll}1 & 0 & 0 & -1 & 0 & d_{1} \sin \left(\theta_{F_{1}}+\phi_{1}\right) & 0 & 0 & 0 & \ldots & 0 & 0 & 0\end{array}\right)$

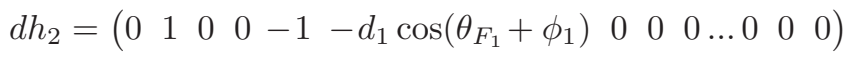

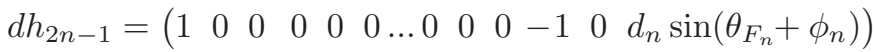

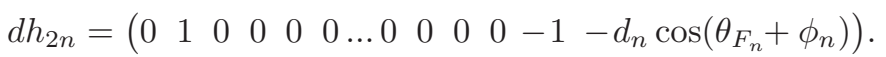
and therefore $\Delta^{\star}$ is given by (15). $\operatorname{dim}\left(\Delta^{\star}\right)=n+3$ and that $\Delta^{\star}$ and $G$ are nonsingular in $\mathcal{X}$.

Note that condition (11) is satisfied but (7) is not. Therefore the problem is solvable if and only if the vector $\left(v_{L} \omega_{L}\right)^{T}$ can be measured.

Control (10) with $\alpha(x)$ and $\beta(x)$ given in (8) and $\gamma(x)$ in (12) is the solution of the formation control problem. Since system (16)-(17) has stable internal dynamics [5], asymptotic stability is guaranteed by choosing $\mu$ according to (9).

Remark 1: Matrices (13) and (14) have a scalable structure. Therefore if a new follower joined the formation it would only need to add two new $2 \times 2$ matrices to $\beta(x)$ and $\gamma(x)$.

\section{SIMULATION RESULTS}

Figure 2 shows the results of three simulation experiments we conducted to validate the state feedback controller

TABLE I

LEADER VELOCITY VECTOR AND PARAMETERS $d_{i}, \phi_{i}$.

\begin{tabular}{|c|c|c|c|}
\hline Trajectory & Rectilinear & Circular & Sinusoidal \\
\hline$v_{L}(t)[\mathrm{m} / \mathrm{s}]$ & 1.5 & 2.1 & 2 \\
\hline$\omega_{L}(t)[\mathrm{rad} / \mathrm{s}]$ & 0 & $\pi / 6$ & $-\sin (1.1 t)$ \\
\hline$d_{1}, d_{2}[\mathrm{~m}]$ & 0.5 & 0.5 & 0.5 \\
\hline$d_{3}, d_{4}[\mathrm{~m}]$ & 1 & 1 & 1 \\
\hline$\phi_{1}, \phi_{3}[\mathrm{rad}]$ & $-\pi / 3$ & $-\pi / 3$ & $-\pi / 3$ \\
\hline$\phi_{2}, \phi_{4}[\mathrm{rad}]$ & $\pi / 3$ & $\pi / 3$ & $\pi / 3$ \\
\hline
\end{tabular}


TABLE II

INITIAL CONDITIONS OF THE LEADER AND THE FOLLOWERS.

\begin{tabular}{|c|c|c|c|c|c|c|c|c|c|}
\hline Trajectory & \multicolumn{3}{|c|}{ Rectilinear } & \multicolumn{3}{c|}{ Circular } & \multicolumn{3}{c|}{ Sinusoidal } \\
\hline & $x(0)[\mathrm{m}]$ & $y(0)[\mathrm{m}]$ & $\theta(0)[\mathrm{rad}]$ & $x(0)[\mathrm{m}]$ & $y(0)[\mathrm{m}]$ & $\theta(0)[\mathrm{rad}]$ & $x(0)[\mathrm{m}]$ & $y(0)[\mathrm{m}]$ & $\theta(0)[\mathrm{rad}]$ \\
\hline leader & 5 & 2 & $\pi / 4$ & 5 & 2 & $\pi / 2$ & 5 & 2 & $\pi / 2$ \\
\hline follower 1 & 3 & 1 & $5 \pi / 6$ & 3 & 0 & $3 \pi / 4$ & 3 & 1 & $5 \pi / 6$ \\
\hline follower 2 & 5 & -0.5 & $\pi / 2$ & 6.2 & -1 & $\pi / 4$ & 6 & 0 & $\pi / 4$ \\
\hline follower 3 & 3.5 & 4 & 0 & 1 & 1 & $\pi / 8$ & 3.5 & 5 & 0 \\
\hline follower 4 & 7.2 & 1 & 0 & 7.2 & 1 & $\pi / 3$ & 7 & 1.2 & $\pi / 8$ \\
\hline
\end{tabular}

designed in Sect. IV. The formation consists of four followers.

The velocity vector $\left(v_{L} \omega_{L}\right)^{T}$ and parameters $d_{i}, \phi_{i}$ for the three trajectories, rectilinear, circular and sinusoidal, are given in Table I. With these parameters the robots travel in a wedge-like formation at steady state. The initial conditions of the leader and the followers are shown in Table II. We set the gains $\lambda_{1}=\lambda_{2}=\ldots=\lambda_{8}=1.2$.

Figs. 2(a),(c),(e) show the path of the five robots in the three simulations. In order to have a temporal reference in the figures the robots are drawn each second. Figs. 2(b),(d),(f) show the corresponding output vectors $y(x)$ (in Figs. 2(b),(d),(f) we followed the same colors convention used in Figs. 2(a),(c),(e)). The outputs are zeroed after about 4 seconds. After this time period the robots correctly keep the formation.

\section{CONCLUSIONS AND FUTURE WORKS}

The original contribution of the paper is to present the leader-follower formation control of nonholonomic mobile robots as a disturbance decoupling problem. By means of purely geometrical conditions, we show that the formation control problem is solvable if and only if the velocity vector of the leader can be measured. A scalable solution (with the number of followers) to this problem is also provided. The main idea of the paper is to interpret leader control input as a disturbance affecting the state of the leader-follower dynamic system. Extensive simulation experiments prove the effectiveness of the proposed approach.

Future research lines include the extension of our results to formations with saturations on the control inputs and the study of more general multirobot formations, e.g. hierarchical formations described by rooted tree graphs [6].

\section{REFERENCES}

[1] T. Balch and R.C. Arkin. Behavior-based formation control for multirobot teams. IEEE Transactions on Robotics and Automation, 14(6):926-939, 1998.

[2] G. Basile and G. Marro. Controlled and Conditioned Invariant Subspaces in Linear System Theory. Journal of Optimization Theory and Applications, 3(5):305-315, 1969.
[3] G. Basile and G. Marro. Controlled and Conditioned Invariants in Linear System Theory. Prentice Hall, Englewood Cliffs, New Jersey, 1992.

[4] L.E. Buzogany, M. Pachter, and J.J. D’Azzo. Automated Control of Aircraft in Formation Flight. Proc. AIAA Guidance, Navigation, and Control Conference, pages 1349-1370, 1993.

[5] L. Consolini, F. Morbidi, D. Prattichizzo, and M. Tosques. On the Control of a Leader-Follower Formation of Nonholonomic Mobile Robots. In Proc. of the 45th IEEE Conference on Decision and Control, pages 5992-5997, 2006.

[6] L. Consolini, F. Morbidi, D. Prattichizzo, and M. Tosques. A Geometric Characterization of Leader-Follower Formation Control. In Proc. IEEE International Conference on Robotics and Automation, 2007, to appear.

[7] A.K. Das, R. Fierro, V. Kumar, J.P. Ostrowsky, J. Spletzer, and C. Taylor. A vision-based formation control framework. IEEE Trasaction on Robotics and Automation, 18(5):813-825, 2002.

[8] D.B. Edwards, T.A. Bean, D.L. Odell, and M.J. Anderson. A leaderfollower algorithm for multiple AUV formations. In Proc. of IEEE/OES Autonomous Underwater Vehicles, pages 40-46, 2004.

[9] R. Fierro, C. Belta, J.P. Desai, and V. Kumar. On controlling aircraft formations. In Proc. of the 40th IEEE Conference on Decision and Control, volume 2, pages 1065-1070, 2001.

[10] T.I. Fossen. Guidance and Control of Ocean Vehicles. John Wiley \& Sons, 1994.

[11] F. Giulietti, L. Pollini, and M. Innocenti. Autonomous formation flight. IEEE Control Systems Magazine, 20(6):34-44, 2000.

[12] A. Isidori. Nonlinear Control Systems. Springer, 3rd edition, 1995.

[13] A. Isidori, A.J. Krener, C. Gori-Giorgi, and S. Monaco. Nonlinear Decoupling via Feedback: A Differential Geometric Approach. IEEE Transactions on Automatic Control, 26(2):331-345, 1981.

[14] T.J. Koo and S.M. Shahruz. Formation of a group of unmanned aerial vehicles (UAVs). In Proc. of the American Control Conference, volume 1, pages 69-74, 2001.

[15] J.R. Lawton, R.W. Beard, and B.J. Young. A Decentralized Approach to Formation Maneuvers. IEEE Transactions on Robotic and Automation, 19(6):933-941, 2003.

[16] M.A. Lewis and K.H. Tan. High Precision Formation Control of Mobile Robots Using Virtual Structures. Autonomous Robots, 4(4):387-403, 1997.

[17] Z. Lin, B.A. Francis, and M. Maggiore. Necessary and Sufficient Graphical Conditions for Formation Control of Unicycles. IEEE Transactions on Automatic Control, 50(1):121-127, 2005.

[18] G.L. Mariottini, G.J. Pappas, D. Prattichizzo, and K. Daniilidis. Vision-based localization of leader-follower formations. In Proc. of the 44th IEEE Conference on Decision and Control, pages 635-640, 2005.

[19] H. Nijmeijer and A. van der Schaft. Controlled Invariance for Nonlinear Systems. IEEE Transactions on Automatic Control, 27(4):904-914, 1982.

[20] H. Nijmeijer and A. van der Schaft. The Disturbance Decoupling Problem for Nonlinear Control Systems. IEEE Transactions on Automatic Control, 28(5):621-623, 1983.

[21] W.A. van den Broek and J.M. Schumacher. Noncooperative disturbance decoupling. Systems \& Control Letters, 41(5):361-365, 2000.

[22] W.M. Wonham and A.S. Morse. Decoupling and pole assignment in linear multivariable systems: A geometric approach. SIAM Journal of Control, 8(1):1-18, 1970. 
TuC08.4

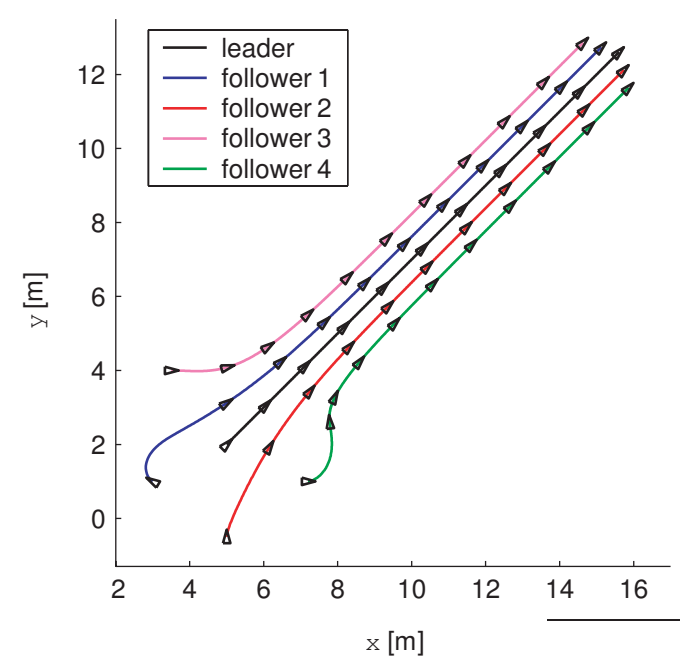

(a)

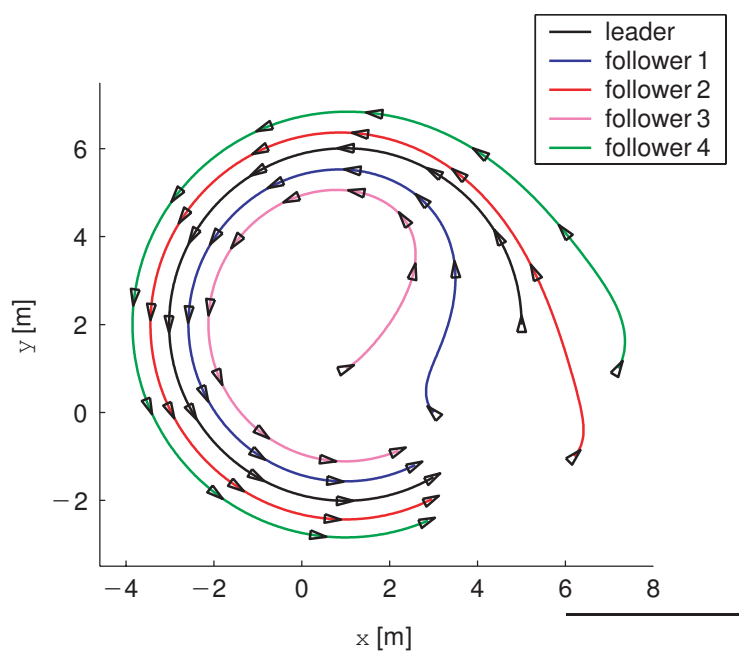

(c)

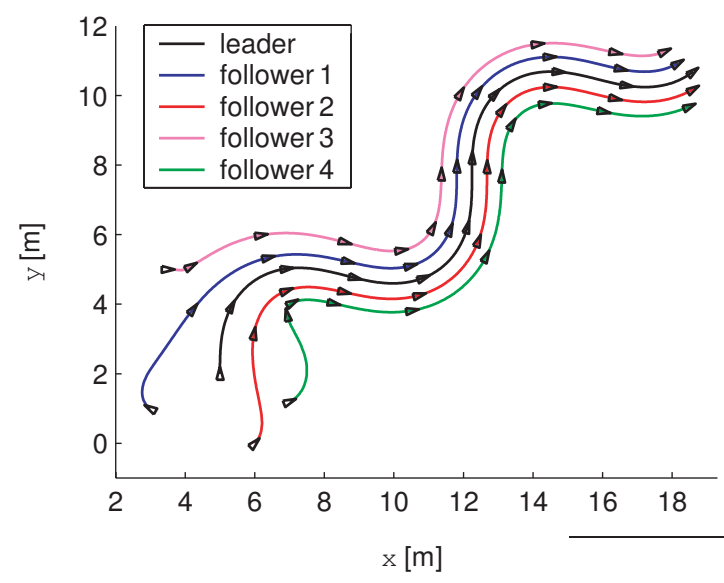

(e)

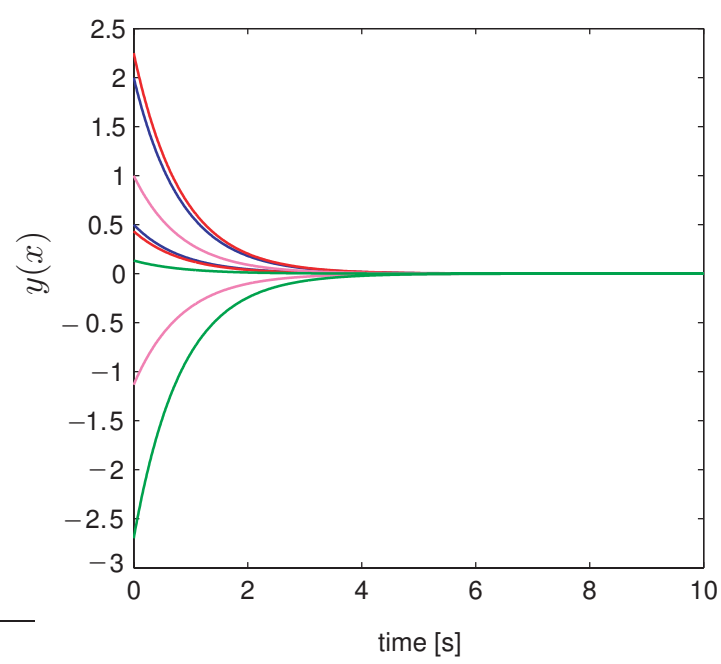

(b)

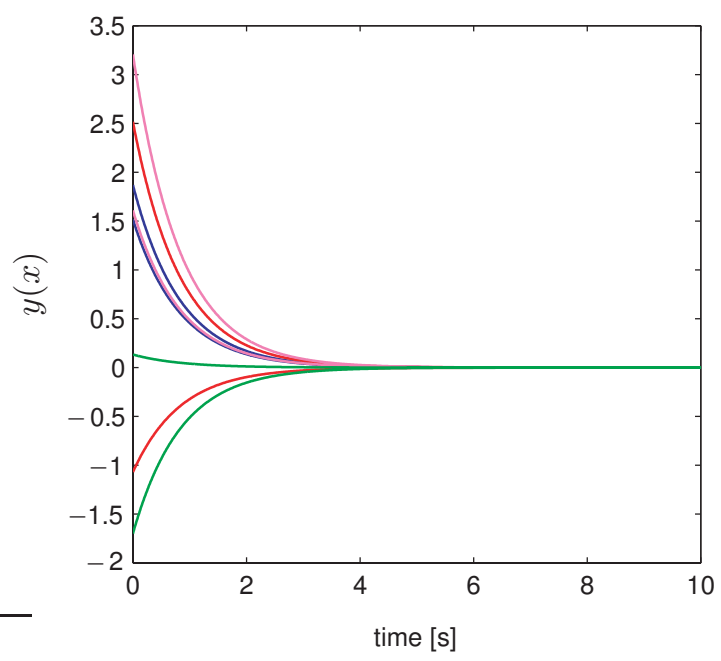

(d)

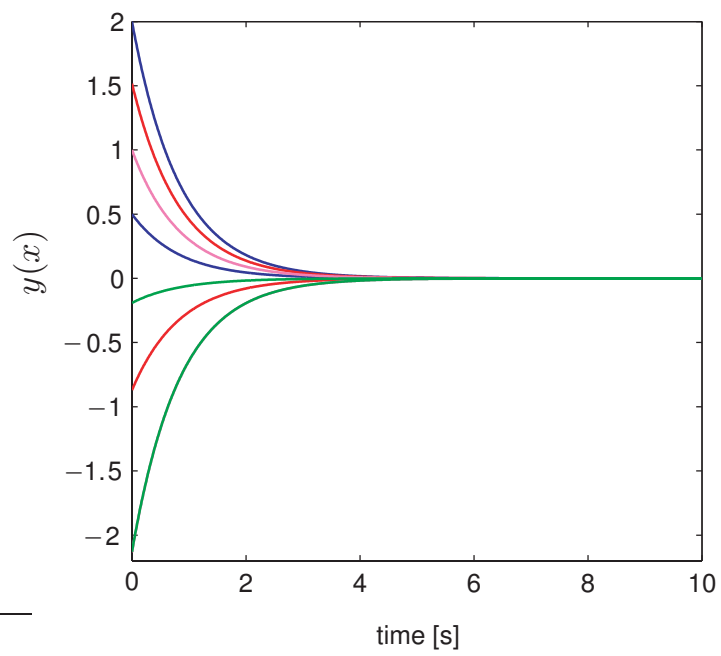

(f)

Fig. 2. (a) Rectilinear trajectory, (c) circular trajectory, (e) sinusoidal trajectory; (b),(d),(f) the corresponding output vectors $y(x)$. 